\title{
El impacto de la evaluación educativa en el desarrollo de competencias en la universidad. La perspectiva de las primeras promociones de graduados
}

\author{
Entidad que Financia: Plan Nacional I+D+I.
}

Ministerio de Economía y Competitividad.

Duración: 3 años (2013-2015)

\section{Resumen de la investigación}

El proyecto impulsado por la Universitat de Barcelona pretende analizar el impacto de la evaluación educativa en el desarrollo de competencias en la universidad a partir de la percepción de las primeras promociones de graduados formados en el marco del EEES.

La finalidad es recoger información acerca del impacto que ha tenido la implementación de los grados universitarios sobre las competencias adquiridas por los estudiantes a partir de la percepción de éstos sobre sus propias competencias y de la opinión de los profesores y los empleadores sobre las competencias que consideran necesarias para el ejercicio profesional.

Por ello se pretende recoger, a través de cuestionarios, la opinión de los graduados y su evolución durante los primeros años después de su graduación. Por otra parte, se desea explorar a partir de entrevistas, buenas prácticas que se estén realizando y que permitan, efectivamente, acreditar competencias tanto específicas (CE) como transversales (CT), en especial aquéllas en las que el profesorado y empleadores hallen más consenso. Finalmente, se desea ofrecer el conocimiento recogido y el repositorio de instrumentos que faciliten el análisis de dicha evolución en un espacio en línea con herramientas abiertas.

La investigación parte fundamentalmente de los resultados de los estudios: uno, el proyecto REFLEX (http://www.fdewb.unimaas.nl/roa/reflex/) en cuyas conclusiones destaca la importancia de equilibrar las actividades para el desarrollo de las 
competencias profesionales con enseñanzas de carácter más académico y el hecho de que no sólo se debe prestar atención al diseño curricular y a los métodos de enseñanza, sino sobre todo a la evaluación. Otro, los realizados por la Agencia para la Calidad del Sistema universitario catalán, AQU, (http://www.aqu.cat/estudis/index_es.html\#.U57EcJTV9rQ), sobre la inserción laboral de la población graduada de las universidades catalanas que constatan un cierto desfase entre las competencias requeridas (solución de problemas, toma de decisiones, capacidad de gestión...) y aquéllas en las que el estudiante se ha formado. Finalmente se recogen la baja valoración que los egresados hacen de la formación práctica que se proporciona en las universidades.

Esta investigación aporta varias novedades sustantivas: se realiza con nuevos graduados, analiza la evolución de la percepción de dichos graduados (con un estudio longitudinal de tres años), focaliza la atención en la importancia de los procesos de evaluación en el desarrollo de las competencias, pretende identificar las competencias y ahondar en la comprensión de los procesos de atribución, indaga en los procesos de evaluación formativa, evaluación final o acreditativa de las competencias de grado y pretende contrastar la información obtenida para hallar los principales puntos de coincidencia y divergencia entre estudiantes, profesorado y empleadores para ofrecer pautas que mejoren las actuales propuestas curriculares y orienten los futuros diseños.

\section{Objetivos}

1. Caracterizar la situación actual en la adquisición de competencias. Realizar un diagnóstico de la primera promoción de graduados bajo el EEES.

2. Comprender las características, tipos de procesos e instrumentos asociados a la evaluación que promueve el desarrollo de competencias.

3. Estudiar la evolución en las percepciones de los graduados a lo largo del tiempo.

4. Contrastar la perspectiva a lo largo del tiempo de los egresados con la percepción de profesorado y empleadores respecto a las competencias y al papel de la evolución en su desarrollo.

5. Identificar buenas prácticas de evaluación sumativa del logro de competencias al final de las titulaciones.

6. Crear y alojar, en un espacio virtual abierto, protocolos de evaluación de competencias adaptables para diversas titulaciones.

El impacto de los resultados del proyecto se articulará en torno a una doble vía: los productos derivados del proceso y de los resultados de la investigación para la difusión científica, y la implementación de una plataforma o espacio virtual de uso abierto con el repositorio final de respuestas. 


\section{Investigadores}

\section{Investigadora principal}

Dra. Elena Cano. Universitat de Barcelona.

\section{Investigadora coordinadora en la URV}

Dra. Juana María Tierno García.

\section{Investigadoras de la URV}

Dra. Charo Barrios Arós

Dra. Pilar Iranzo García

Marta Camarero Figuerola (colaboradora)

\section{Otras universidades investigadoras}

Universitat Autònoma de Barcelona, Universidad de Granada, Universitat de les Illes Balears, Universitat Oberta de Catalunya, Universitat Politècnica de Barcelona. 